\title{
Evaluation of Therapeutic Results of A Series of Moroccan Patients Aged 20 To 60 Years Treated According To The Acute Myeloid Leukaemia 03 Protocol At The Paediatric Haematology Oncology Centre In Casablanca
}

Marielle IGALA ( $\sim$ marieligalase@yahoo.fr )

Department of heamatology of university hospital of Casablanca, Morocco

Mouna LAMCHAHAB

Department of heamatology of university hospital of Casablanca, Morocco

Siham CHERKAOUI

Department of heamatology of university hospital of Casablanca, Morocco

Nisrine Khoubila

Department of heamatology of university hospital of Casablanca, Morocco

Bouchra OUKKACHE

Laboratory of heamatology of university hospital of Casablanca, Morocco

Nezha HDA

Cytogenetic laboratory of Casablanca, Morocco

Fatima BACHIR

Hygien Institut of Rabat, Morocco

Meryem QACHOUH

Department of heamatology of university hospital of Casablanca, Morocco

Mohamed RACHID

Department of heamatology of university hospital of Casablanca, Morocco

Abdellah MADANI

Department of heamatology of university hospital of Casablanca, Morocco

Asma QUESSAR

Department of heamatology of university hospital of Casablanca, Morocco

\section{Case Report}

Keywords: acute, myeloblastic, leukemia, AML03 protocole

Posted Date: April 16th, 2021 
DOl: https://doi.org/10.21203/rs.3.rs-409672/v1

License: (c) (1) This work is licensed under a Creative Commons Attribution 4.0 International License. Read Full License 


\section{Abstract}

Acute Myeloblastic Leukaemia (AML) is a malignant haemopathy characterized by infiltration and accumulation in the bone marrow by myeloblastic-type blast cells causing medullary insufficiency. At the global level, the search for improved AML treatment is a long-standing concern.

\section{Purpose}

To evaluate a new protocol AML MA 2003 its therapeutic results and tolerance in de novo AML patients.

\section{Methods}

It was a prospective and descriptive unicentre study carried out from January 2003 to December 2010. It included adults of 20 to 60 years with a diagnosis of de novo AML except a promyelocytic leukemia and without treatment by hydroxyurea.

\section{Results}

A total of 962 AMLs were diagnosed, 438 met the inclusion criteria, $48.4 \%$ women and $51.6 \%$ men with a median age of 39.8 years. $36.1 \%$ received hydroxyurea in pre-induction. $96.3 \%$ patients were induced, 76 $(18 \%)$ died. A total of $178(40.6 \%)$ of the 438 evaluable patients reached the maintenance phase. The overall survival of all patients was $32.4 \%$ at 5 years. Survival without event was $17.6 \%$ for all patients.

\section{Conclusion}

The results of this single-center study illustrate the difficulty of treating AML patients despite the use of drugs recognised for their effectiveness in appropriate doses.

\section{Introduction}

Acute Myeloblastic leukaemia (AML) is a malignant blood disease characterised by the infiltration and accumulation in bone marrow of blast cells, type myeloblast, causing bone marrow failure. (1) Their management is not easy. A global search for improved AML treatment is a long-standing concern.

The experience of the Haematology and Paediatric Oncology Service of Casablanca, in the treatment of AML, started in the 1980s and can be surmised in four major therapeutic periods. Before 1985, the attitude varied between abstention and treatment with 6 Mercaptopurine (6MP) (4). In 1989, the treatment used was the French protocol "CHA". The evaluation found a high death rate before and during induction treatment. The AML 6.96 protocol was implemented in 1996. The overall CR rate was $60 \%$, death rate had increased to $14 \%$ and $16 \%$ of patients were in failure. (5)

\section{Aim of this study}


To evaluate a new protocol AML MA 2003 its therapeutic results and tolerance in de novo AML patients on the Paediatric Haematology/Oncology Unit at the Ibn Rochd University Hospital of Casablanca.

\section{Patients And Methods}

From January 2003 to December 2010 on the Paediatric Haematology/Oncology Unit at the University Hospital Casablanca, patients of 20 to 60 years with a diagnosis of de novo AML (outside of acute promyelocytic leukaemia) untreated (except for the administration of hydroxyurea (HU) to stem a threatening leukocytosis) without cardiac problems, major liver or kidney failure and positive HIV serology were included. The study responded to the recommendations of the national ethics committee on the use of human data. All patients or their representent signed a consent form after being informed of the development of the protocol.

The diagnosis of AML was selected according to the $D$ of classification of the cooperative group, FrenchAmerican-British (FAB). Imuno-phenotyping and cytogenetic analysis, were performed at diagnosis in medullary sampling. According to the World Health Organization (WHO) classification patients were on favourable prognosis $(t(8 ; 21)$ or inversion of chromosome 16$)$, unfavourable prognosis (deletions of chromosome $5,7,3 q$ inversion, $11 q$ abnormality of $19 q, t(6 ; 9), t(9 ; 22)$ and the complex karyotype). The group with an intermediate prognosis, corresponded to all other types of karyotypes.

For hyper-leucocytaire forms (WBC> 50,109 / I), as a pre-induction, a cyto-reductive treatment by HU at 50 $\mathrm{mg} / \mathrm{kg} /$ day per day was administered for 4 days. The chemotherapy protocol included an induction for all patients, a bone marrow aspirate evaluation was performed on D15. For patients whose marrow had more than $20 \%$ blasts, intensification was started at D16. For those who had a bone marrow aspirate of less than $20 \%$ blasts, a second aspirate was conducted between D28-D30 after exit from aplasia. The second induction, identical to the first, was started at D30 after aplasia, for patients who had not needed the intensification cure. The two consolidation treatments included patients in complete remission at the end of the 2 courses of induction and catching up. Prophylactic treatment of the Central Nervous System was administered by intra-thecal injections (IT) on day one of each course. The maintenance treatment lasted 18 months. (Figure 1)

For supportive care, patients received antifungal prophylaxis and digestive decontamination. Mucositis was prevented by administrating mouthwashes containing oral fungizone, and blood transfusion. Febrile neutropenia was brought under control by antibiotics.

Treatment responses were based on the revised criteria Cheson et al: defining the complete response, partial and failure. (6) On the periphery, haematological recovery included a neutrophil count $>1.1^{09} / \mathrm{L}$ and a platelet count $>100.1^{09} / \mathrm{I}$. Early and toxic mortality is defined as any death occurring during preinduction, or during the induction phase related to a complication of the disease or toxicity secondary to treatment. Deaths occurring during the consolidation period will be analysed separately. 
Statistical Analyses was conducted using the SPSS software. The overall survival (OS) and event-free survival (SES) were determined according to the Kaplan-Meir method.

\section{Results}

\section{Patient Characteristics}

During the study period, 962 patients were diagnosed, 438 (45.5\%) met the inclusion criteria, of which $48.4 \%$ were women and $51.6 \%$ men. The sex ratio $M / W$ were 1.06 , the median age of 39.8 years (2060 ans). The average time between the onset of signs and diagnosis, then between diagnosis and start of treatment was 67 days respectively (7-300 days) and 20,5 days (1-120 days). Performance index according to ECOG were 1 for $38.1 \%$ patients. White blood cells were between 50000 and 100000 in $18.5 \%$ of cases and over 100,000 in $12.6 \%$ patients. LAM 1 was the most diagnosed in $37.2 \%$ of cases. (Table 1 )

\section{Cytogenetics}

Karyotype was performed in 399/438 (88.1\%) of cases including 12 cases $(3 \%)$ of failed metaphase and $387(97 \%)$ conclusive results: $74(19.1 \%)$ with favourable prognosis group, $58(15 \%)$ poor prognosis and $255(65.9 \%)$ with an intermediate prognosis. (Table 1)

\section{Pre-induction phase}

Patients enrolled in pre-induction were 158/438 (36.1\%). 12 (7.6\%) of them died, $3(1.9 \%)$ were lost to follow-up (LTF) and for 103 (65.2\%) patients this phase allowed a drop of more than $50 \%$ of leukocytes. In the 158 patients included in pre-induction 143 (90.5\%) have received the remainder of the protocol. A total of 280 patients did not receive pre-induction, 1 died before induction. (Fig 2)

422/438 (96.3\%) patients were induced, 76 (18\%) died either before (59/422) or just after (17/422) evaluation while 11 (2.6\%) were LTF before evaluation. This latter found: 171 (40.5\%) Complete Response, 72 (16.4\%) failures, 28 (6.4\%) partial remission (PR) and in 59 (13.5\%) cases bone marrow aspiration was inconclusive on D15 for various reasons. After this evaluation 24 patients were LTF and 1 put under palliative treatment. (Fig 3)

Intensification was performed to 59/438 (13.5\%) patients. At the end of treatment $28 / 59(47.5 \%)$ were in complet response and received consolidation treatment, 10/59 (16.9\%) died, 20/59 (33.9\%) were taken out of protocol. (Fig 3)

266/438 (60.7\%) patients started the second induction, 10/266 (3.75\%) were LTF, 10 (3.75\%) died during treatment, $24(9 \%)$ discontinued the protocol, $3(1.1 \%)$ have directly received maintenance and $218(82 \%)$ were orientated to the consolidation cure. (Fig 3)

\section{Consolidation 1 and 2}


A total of 246/438 (56.2\%) patients started the first consolidation, 218 came from the second induction treatment and 28 from intensification. In total 11patients relapsed, 11 were LTF, 15 died and 18 were orientated to maintenance therapy. (Fig 3)

Only 191/438 (43.6\%) reached the second consolidation after which 4/191 patients died, 1 patient relapsed, 30 were referred for outpatient consultation without maintenance treatment and 156/191 (81.7\%) to maintenance treatment. (Fig 3)

\section{Maintenance}

A total of 178/438 (40.6\%) assessable patients reached the maintenance phase 3 directly from the second induction, 1 from intensification, 18 from the first consolidation, 156 from the second consolidation. (Fig 3)

\section{Discontinuation}

During the protocol 64/438 (14.1\%) patients discontinuated the treatment: 3 during the pre-phase induction, 35 in induction 1, (11 before and 24 after the evaluation), 5 after intensification, 10 after induction 2, 11 after consolidation 1.

\section{Deaths during the protocol}

A total of 128/438 (29.2\%) patients died during treatment: 12/128 (9.4\%) during the pre-induction phase, $84 / 128(65.7 \%)$ during the two inductions, $10 / 128$ (7.8\%) during intensification, 22/128 (18\%) during the first and second consolidations.

Infections were the main cause of deaths encountered 46/128 (36\%). The documented microbial infection concerned 15/46 (32.6\%) patients: 12 during induction 1 (8 invasive fungal infections and 4 bacterial infections).

\section{Overall survival and event-free survival}

Overall survival of all patients was $32.4 \%$ at 5 years. It was established for patients according to their prognosis group and those for which we had no cytogenetic data. It was $30.8 \%$ for the good prognosis group, $32.6 \%$ for the intermediate group and $32.3 \%$ for the poor prognosis group. Event-free survival (EFS) was $17.6 \%$ for all patients. (Fig 3, 4 and 5 )

\section{Discussion}

Improving the treatment of AML patients is a longstanding preoccupation. It has led to the creation of a support service for all AML patients throughout the country. The high cost of care in private facilities, limiting access, brought the major number of patients to the public sector. This study while not multicenter can be regarded as a reflection of the treatment of AML across the country. 
The average age of patients, 39.8 years, was different from what is found in developed countries due to the inversion of the age pyramid between these 2 types of population. This result was close to that of developing countries because of the younger age of the population. $(7,8)$ In recent years many molecular discoveries and therapeutic strategies have been put in place for the treatment of AML and have imposed that cytogenetic tests and molecular biology be carried out before starting chemotherapy. The results are available within 72 hours, at most 5 days after. (9)

This has the effect of delaying by a few days the beginning of treatment, an average of 5 to 8 days with an impact on the complete remission and progression-free survival according to studies. $(10,11)$ Alongside the role of laboratory tests, a higher number of leukocytes and the advanced age of patients will be responsible for prolonging the time of care for patients. (10)

In our study the processing delay causes are different. The time between diagnosis and treatment in hospital, on average 20.5 days, from the results published to date was extended for other reasons. These, though they were not listed, would be dominated by a reduced bed capacity while demand was high. This capacity problem is not only found in developing countries because many of the major Western centers encounter it also. (10)

During the study 64 (15.3\%) patients were lost sight for various reasons: the distance from the treatment center because patients came from all across the country without possibility of residence in Casablanca in inter-treatment, lack of a local health centre capable of managing infections, transfusions or emergencies, limited access to telephone coverage making it difficult for any regular contact with the patient or family, the long time between hospitalisation due to the large number of patients and limited bed capacity. The cost of treatment for patients often from rural areas without health insurance was also an important parameter for discontinuation of treatment especially after the first induction, during which more than half of patients were lost of view. While it is recognised that the cost of treatment of AML is by far the highest compared to other cancers, in many countries the cost is reduced for the patient by the existence of social coverage or entry into research protocols. $(12,13)$

\section{Prephase- induction}

One of the first attitudes at diagnosis of AML is aimed at correcting the immediate complications of which one is hyper-leukocytosis. It is known to be responsible for a poor prognosis and clinical and biologic complications that can be fatal for the patient. Hyper-leukocytosis in AML is characterized by a mortality ranging from 4 to $29 \%$ at diagnosis, a low rate of complete remission and progression free survival. (14) Cyto-reductive strategies are the subject of much debate between leukopheresis and hydroxyurea use. In our protocol hydroxyurea treatment was used in $158(36.1 \%)$ patients. It has resulted in a fall of more than $50 \%$ of white blood cells in $65.2 \%$ (103) cases. However 12 (7.6\%) died. This phase of treatment was useful for patients who were subsequently able to receive chemotherapy.

\section{Inductions}


During our 8-year study we evaluated the response to a new chemotherapy regimen that used doses of ara-c and daunorubicin as recommended in international standards for patients 20 to 60 years with a diagnosis of LAM novo. The first induction treatment administered to patients allowed 171 (40.5\%) CR, which is an improvement on the previous protocol where there were only $36 \%$. The addition of a second induction increased the rate to $76.3 \%$ or 203 patients of 266 who received it, as described in the literature $(60-80 \%)(3,15,16)$. But again, the poor results in the first induction could partly be explained by the early realisation of the bone marrow aspiration at D15 while spinal cord recovery was not effective on that date. This attitude is questionable because conventionally, evaluation is performed between day 21 and day 28 of induction except for some studies that test new agents or use induction treatment intensification like ours. (16)

\section{Intensification}

The intensification was used for all patients whith more than $20 \%$ blasts at the evaluation. It favoured remission of $28(47.5 \%)$ among the 59 patients who received it. They subsequently accessed to the next phase of the protocol.

For our patients, failing to provide a transplant for those whose prognostic factors indicated it, we administered two consolidation treatments which helped to maintain a good response in $82.9 \%$ of patients after the first consolidation and $91.6 \%$ for those who received the second. The international study groups recommend AML patients in clinical and haematological remission be administered several consolidation cycles while knowing that there is no consensus on a post-induction treatment strategy. (17) Today, the choice of consolidation therapy is guided by prognostic factors and includes conventional intensive chemotherapy, maintenance therapy or transplantation. (7) If the number of cycles is controversial, the doses of drugs to be administered are not. $(2,3,8,16,18)$.

Maintenance therapy, unlike acute lymphoblastic leukemia's and pro-myelocytic AML, while it is routinely offered, is not unanimous. (19) It has an interest in the survival of the elderly. (2) In our protocol $40.6 \%$ of patients received maintenance treatment. Our therapeutic attitude was justified by the lack of possibility of intensive treatment by auto or allograft in our unit even if it has been demonstrated that there was no benefit to using it. (16)

During the study, deaths occurred during the induction phase. Infection was the most common cause with a rate of $36 \%$. This figure is certainly underestimated because all the deaths occurred in hospital. Their large numbers during the induction phase could partly be linked to the condition of patients at diagnosis, the lack of anti-infective prophylaxis and especially by the lack of knowledge of the microbial ecosystem of the service that would have helped initiate a probabilistic antimicrobial therapy before the microbial infection evidence. During induction 1 a large number of fungal infections with Candida were found. Jih-Luh Tang et al reported the incidence of invasive fungal infections in the induction phase in patients treated for AML. (20)

The mortality observed in the treatment of AML has led many teams to focus their activities on preventive measures by giving supportive care to reduce deaths from infections and bleeding. Antimicrobial 
prophylaxis and administration of growth factor have allowed this goal to be widely achieved. $(21,22)$

Survival at 5 years for $32.4 \%$ among all the patients in our study was well below the standards of $40-50 \%$ usually described for this age. (3) This result is explained by the large number of deaths $(128 ; 29.2 \%)$ throughout the treatment but even more so during the induction phase. Despite relatively different values for the overall 5-year survival between favorable and intermediate prognosis, we note that the results for the two groups were virtually superimposed curves. This leaves us to suppose the existence of interconnections between these two groups that only cytogenetics could establish. Molecular biology analyses should hold all their importance for a more refined prognosis classification for patients and to help us take the appropriate therapeutic action. The international study groups now recommend a number of routine tests, including molecular biology for the prognostic evaluation of AML. (23)

\section{Conclusion}

The results of this single-center study illustrate the difficulty of treating AML patients despite the use of drugs recognised for their effectiveness in appropriate doses. The cost of treatment, limited hospital accommodation capacity, the absence of similar structures in the country, and the lack of effective support care are all reasons that contributed to the abandonment of treatment, death and failure. Hopes are raised following the construction of new structures throughout the country, reducing exam and medicines costs which are no longer paid for by patients, efforts in microbial identification and treatment of febrile neutropenia suitable for the microbial ecosystem of the service.

\section{Declarations}

\section{Acknowledgments}

Thanks to Patricia Delia and all staff of the Hematology and Pediatric Oncology Department of Casablanca University Hospital for their support throughout this work

\section{Conflit of interest}

The authors declare don't have any conflict of interest

\section{References}

1- WEINSTEIN JH. Acute Myeloid Leukemia. In Chilhood leukemias, edited by Ching Hong Pui. Cambridge university press $2000 ; 322-335$.

2- Rowe Jacob M. Optimal induction and post-remission therapy for AML in first remission. Hematology 2009; 1: 396-405.

3- Roboz Gail J. Novel approches to the treatment of acute myeloid leukemia. Hematology 2001; 1: 43-50. 
4- LAZRAK M. Les leucémies aiguës myéloblastiques. Thèse-Casablanca, nº 125, pp 66, 1983

5- QACHOUH M, Quessar A, Harif M, Benchekroun S. Leucémies aiguës myéloblastiques de l'adulte : Evaluation du protocole AML. 6.96. Tunisie médicale 2003;81(7): 461-5.

6- Cheson BD, Bennett JM, Kopecky KJ, Büchner T, Willman CL, Estey EH. Revised recommendations of the International Working Group for Diagnosis, Standardization of Response Criteria, Treatment Outcomes, and Reporting Standards for Therapeutic Trials in Acute Myeloid Leukemia. J Clin Oncol. 2004; 22(3):576.

7- Philip C, George B, Ganapule A, Korula A, Jain P, Alex AA, et al. Acute myeloid leukaemia: challenges and real world data from India. Br J Haematol. 2015 Jul;170(1):110-7.

8- Azevedo MC, Velloso EDRP, Bucheri V, Chamone DAF, Dorlhiac-Llacer PE. Possible benefit of consolidation therapy with high dose-cytarabine on overall survival of adults with non-promyelocytic myeloid leukemia. Braz J Med Res 2015; 48(2): 178-85

9- Ostgard LS, Nørgaard JM, Sengeløv H, Holm MS, Jensen MK, Kallenbach M, Marcher CW et al. Impact of chemotherapy delay on short- and long-term survival in younger and older AML patients: a Danish population-based cohort study. Leukemia. 2014; 28(9):1926-9.

10- Bertoli S, Bérard E, Huguet F, Huynh A, Tavitian S, Vergez F. Time from diagnosis to intensive chemotherapy initiation does not adversely impact the outcome of patients with acute myeloid leukemia. Blood. 2013; 121(14):2618-26.

11-Sekeres MA, Elson P, Kalaycio ME, Advani AS, Copelan EA, Faderl S. Time from diagnosis to treatment initiation predicts survival in younger, but not older, acute myeloid leukemia patients. Blood. 2009; 113(1):28-36.

12- Wang HI, Aas E, Howell D, Roman E, Patmore R, Jack A et al. Long-term medical costs and life expectancy of acute myeloid leukemia: a probabilistic decision model. Value Health. 2014; 17(2): 205-14.

13-Zeidan AM, Mahmoud D, Kucmin-Bemelmans IT, Alleman CJ, Hensen M, Skikne B et al. Economic burden associated with acute myeloid leukemia treatment. Expert Rev Hematol. 2016; 9(1):79-89.

14- Kuo KH, Callum JL, Panzarella T, Jacks LM, Brandwein J, Crump M. A retrospective observational study of leucoreductive strategies to manage patients with acute myeloid leukaemia presenting with hyperleucocytosis. Br J Haematol. 2015; 168(3): 384-94.

15- Ryting M, Ravandi F, Estey E, Cortes J, Faderl S, Garcia-Manero G et al. Intensively timed combinaison chemotherapy for induction of adult patients with acute myeloid leukemia. Cancer 2010: 5272-7.

16- Döhner Hartmut, Estey Elihu H, Amadori S, Appelbum Frederick R, Büchner T, Burnett Alan K. Diagnosis and management of acute myeloid leukemia in adults: recommandations from an 
international expert panel, on behalf of the European LeukemiaNet. Blood 2010; 115(3): 453-74.

17- Fey M, Dreyling M. Acute myeloblastic leukemia in adult patients: ESMO clinical Recommandations For diagnosis, treatment and follow-up. Annals of oncology 2009; 20(4): 100-1.

18- Löwenberg. Sense and nonsense of high-dose cytarabine for acute myeloid leukemia. Blood 2013; 121(1): 26-8.

19- Perel Y, Auvrignon A, Leblanc T, Vannier JP, Michel G, Nelken B. Impact of addition of maintenance therapy to intensive induction and consolidation chemotherapy for childhood acute myeloblastic leukemia: results of a prospective randomized trial, LAME 89/91. Leucámie Aiqüe Myéloïde Enfant. J Clin Oncol 2002 Jun 15; 20(12): 2774-82

\section{Tables}

Table 1 Clinical and demographic characteristic of the patients 


\begin{tabular}{|c|c|}
\hline Variable & $\mathrm{n}(\%)$ \\
\hline Age $(n=438)$ & 39.8 ans \\
\hline \multicolumn{2}{|l|}{ Gender } \\
\hline Male & $226(51.6 \%)$ \\
\hline Female & $212(48.4 \%)$ \\
\hline Ratio M/F & 1.06 \\
\hline Diagnostic delay & $67 \mathrm{j}(7-300 \mathrm{j}$ \\
\hline Delay diagnostic-treatment & $20.5 \mathrm{j}(1-120)$ \\
\hline \multicolumn{2}{|l|}{ FAB* classification $(n=438)$} \\
\hline MO & $19(4.3 \%)$ \\
\hline M1 & $163(37.2 \%)$ \\
\hline M2 & $123(28.1 \%)$ \\
\hline M4 & $44(10 \%)$ \\
\hline M5 & $34(7.8 \%)$ \\
\hline M6 & $16(3.7 \%)$ \\
\hline M7 & $1(0.2 \%)$ \\
\hline Not classified & $38(8.7 \%)$ \\
\hline \multicolumn{2}{|l|}{ ECOG* PS* $(n=438)$} \\
\hline 0 & $146(33.3 \%)$ \\
\hline 1 & $167(38.1 \%)$ \\
\hline 2 & $61(13.9 \%)$ \\
\hline 3 & $38(8.7 \%)$ \\
\hline 4 & $26(5.9 \%)$ \\
\hline Karyotype(n=387) & $74(19.1 \%)$ \\
\hline Favorable & $255(65.9 \%)$ \\
\hline Intermediate & $58(15 \%)$ \\
\hline Unfavorable & $12(3 \%)$ \\
\hline
\end{tabular}


Failed

White blood cell: Giga/l $(n=438)$

$0-49000$

$302(68.9 \%)$

50000-100000

$>100000$

$55(12.6 \%)$

FAB : French American British

ECOG : Eastern Cooperative Oncology Group

PS : Performans status

Figures

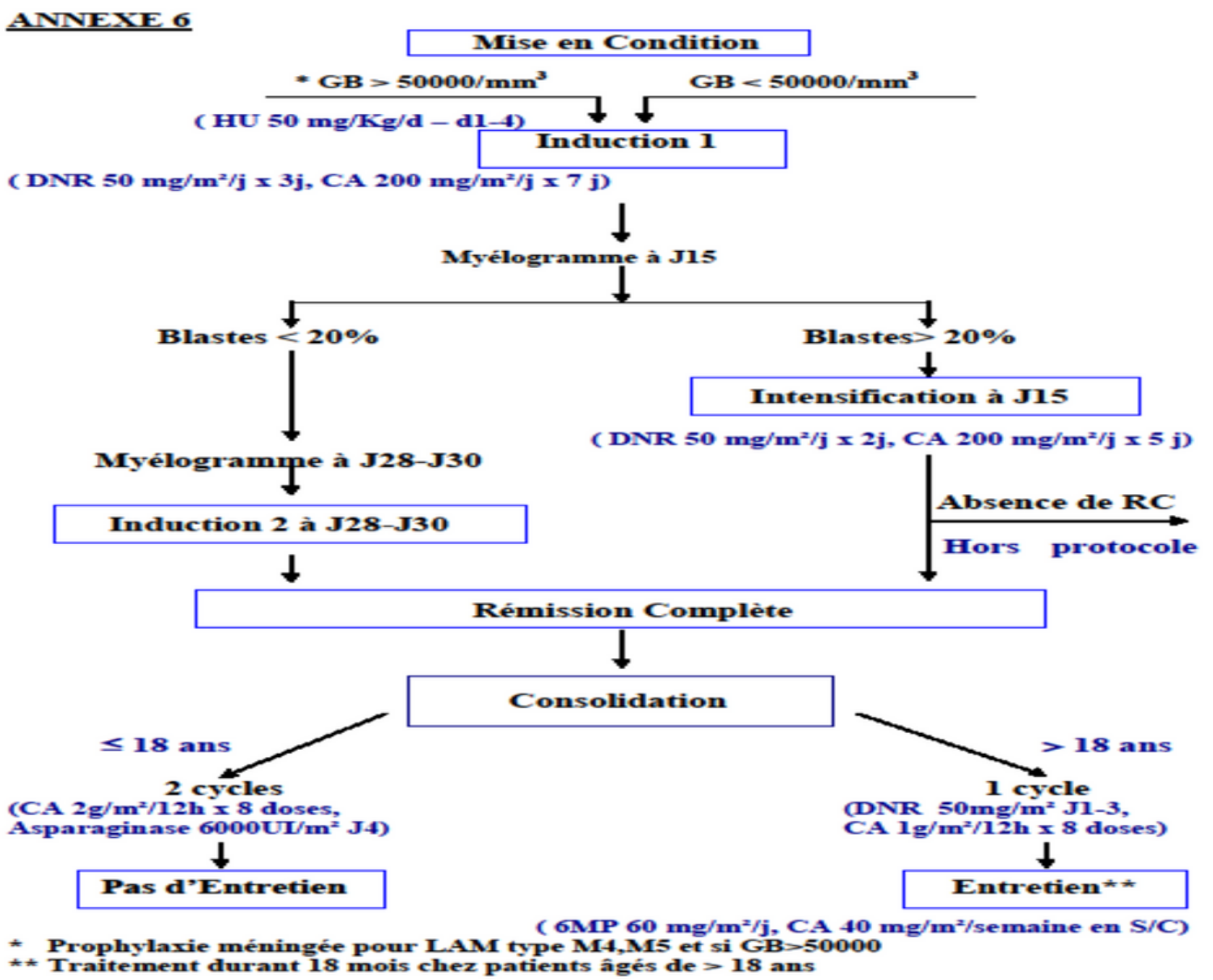

Figure 1 
438 Patients assessables

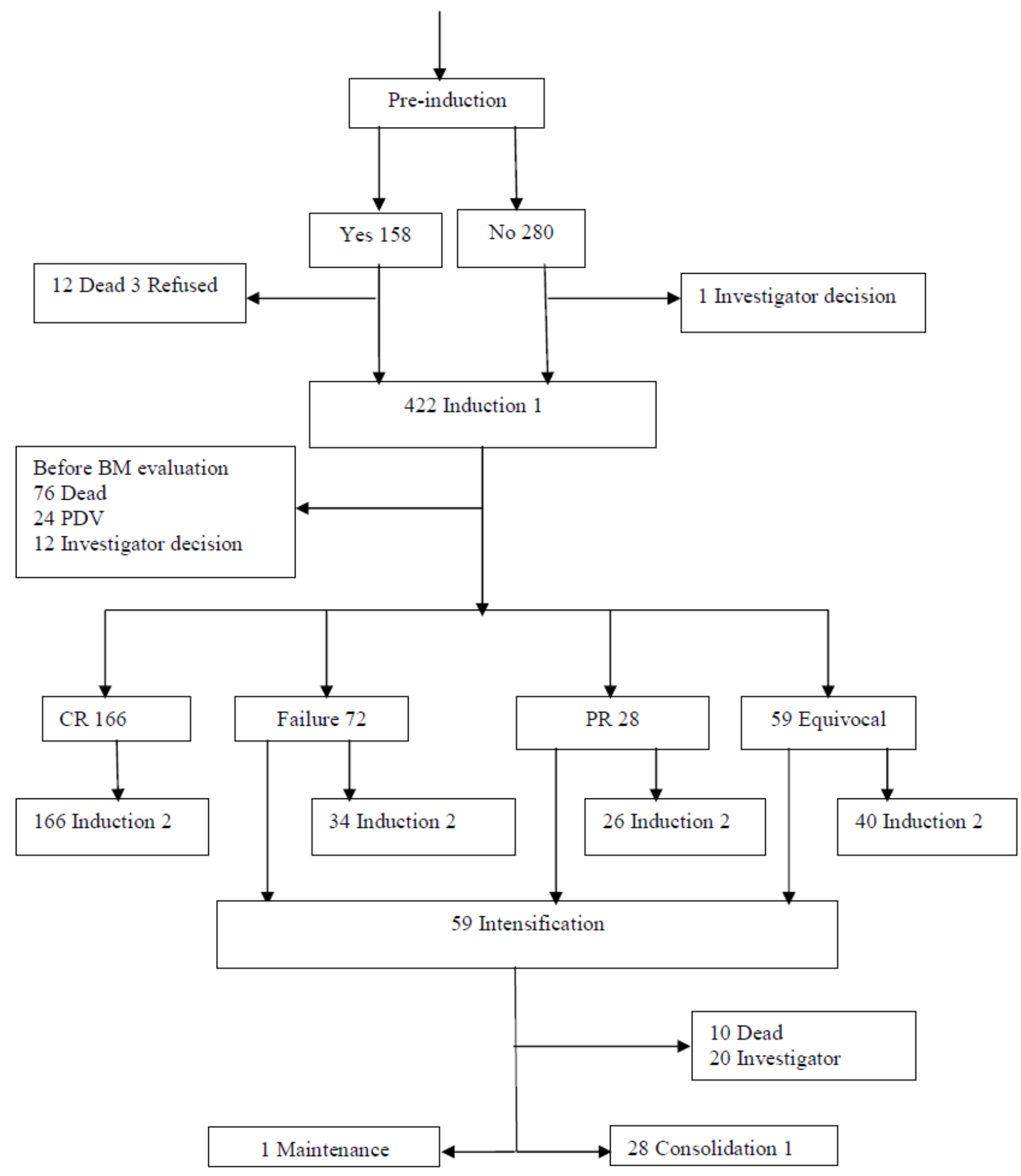

Figure 2

Profile of the study and outcome of patients after pre-induction induction and intensification BM: Bone Marrow; CR: Complete Remission; PR : Partial Response; Intensive: Intensification 


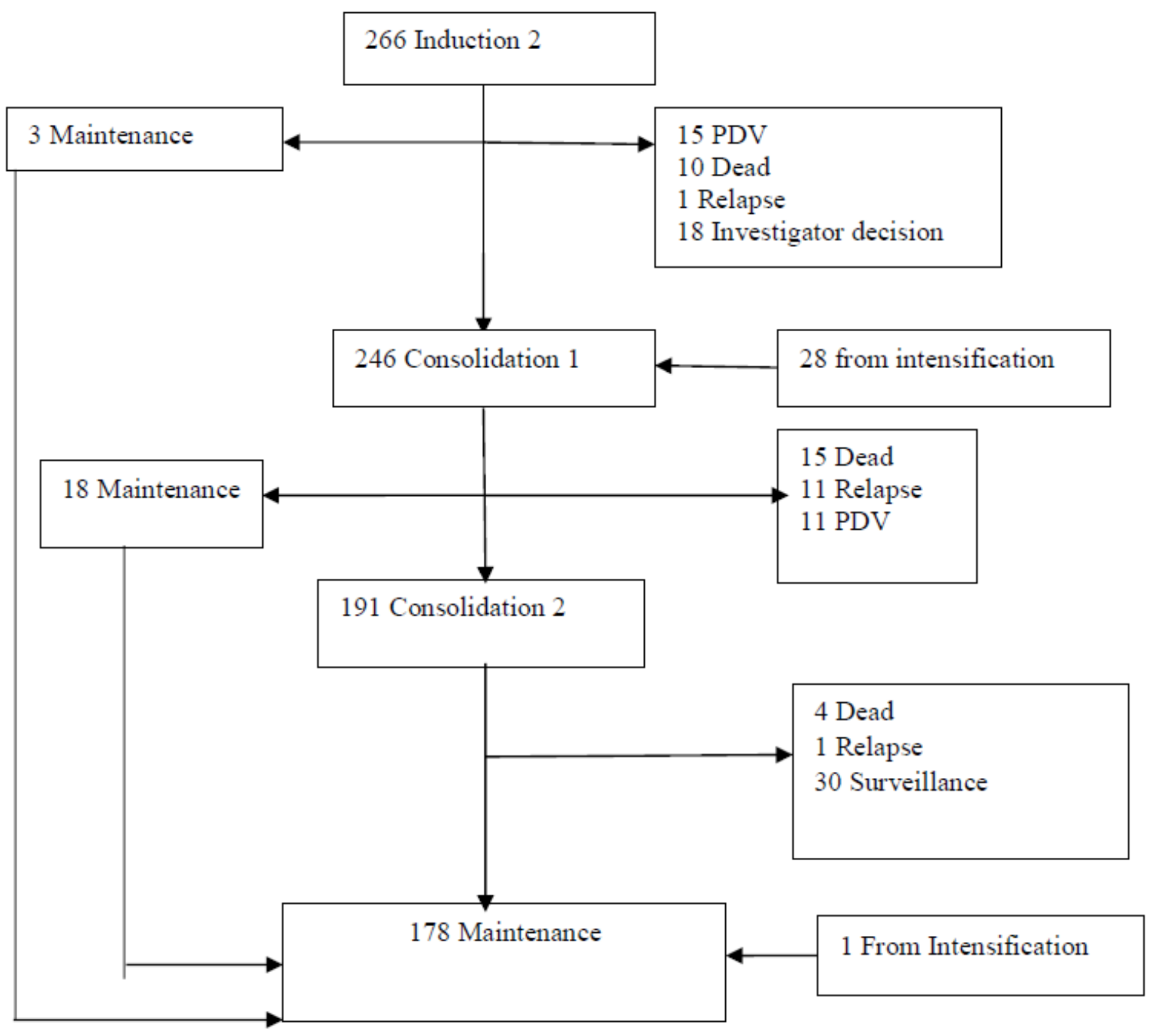

Figure 3

Outcom of patients after Induction 2, Consolidation 1, 2 and Maintenance therapy 


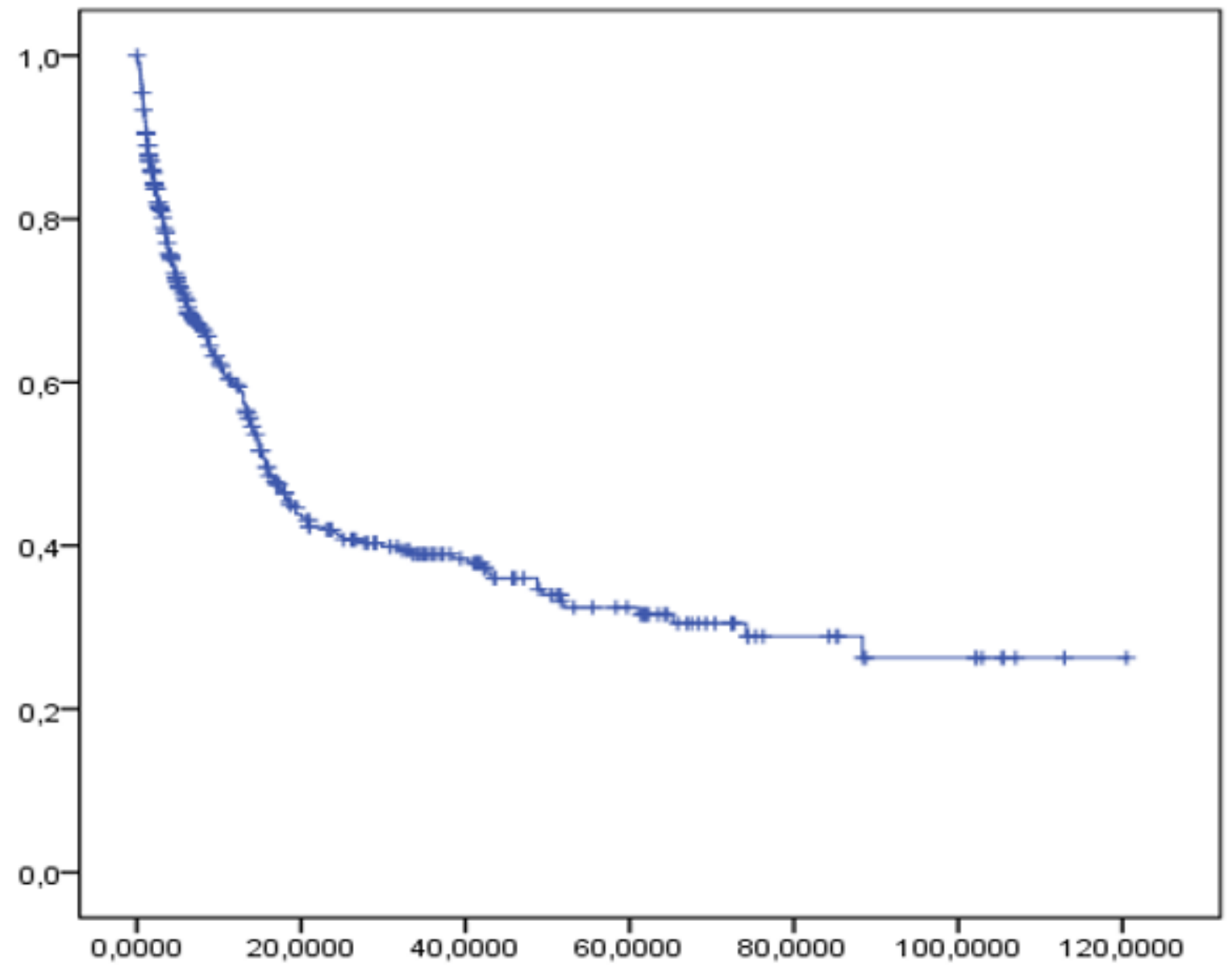

Figure 4

Global survival at 5 years of patients treated by the AML protocol 03 


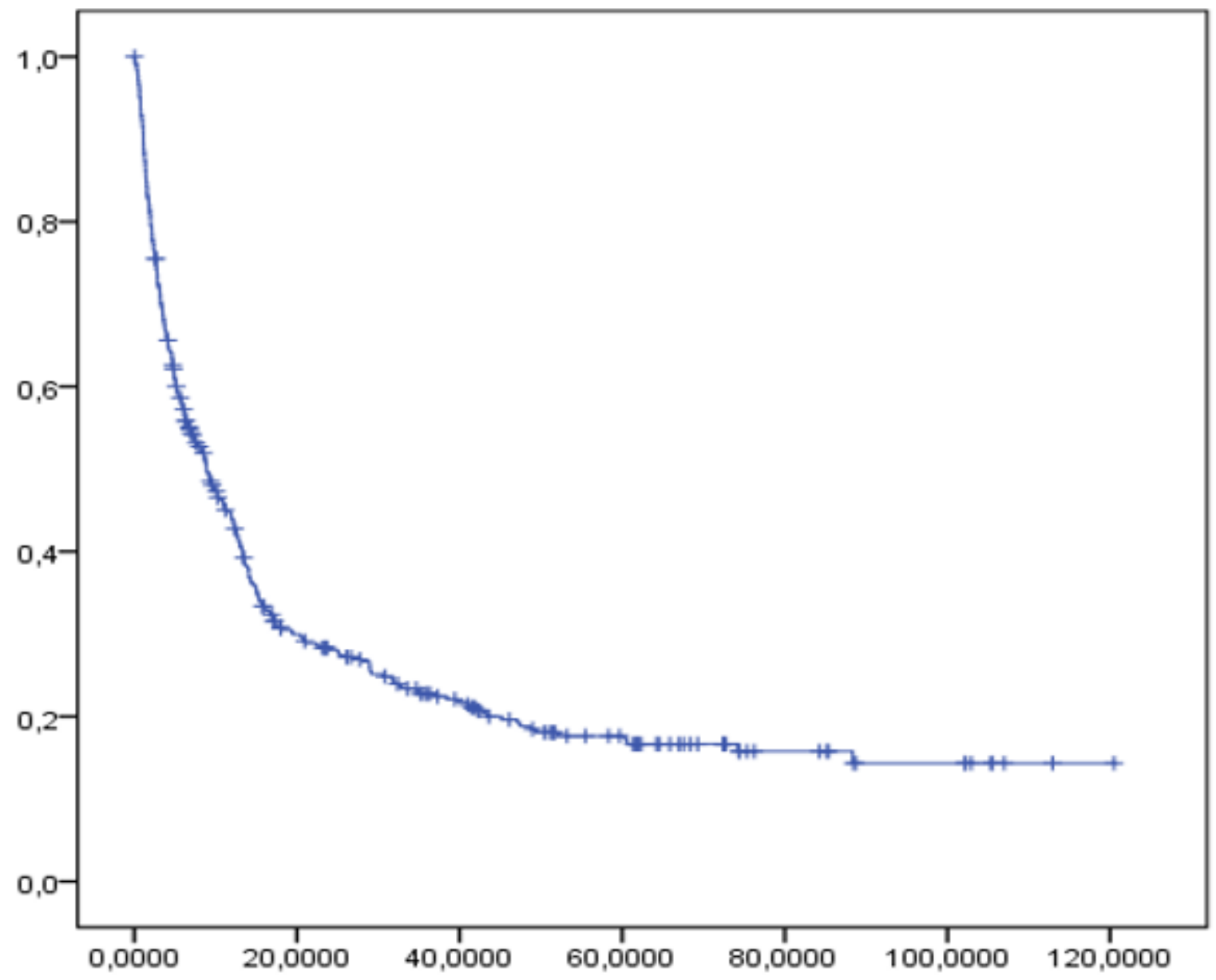

Figure 5

Survival without incident of patients treated by the AML protocol 03 


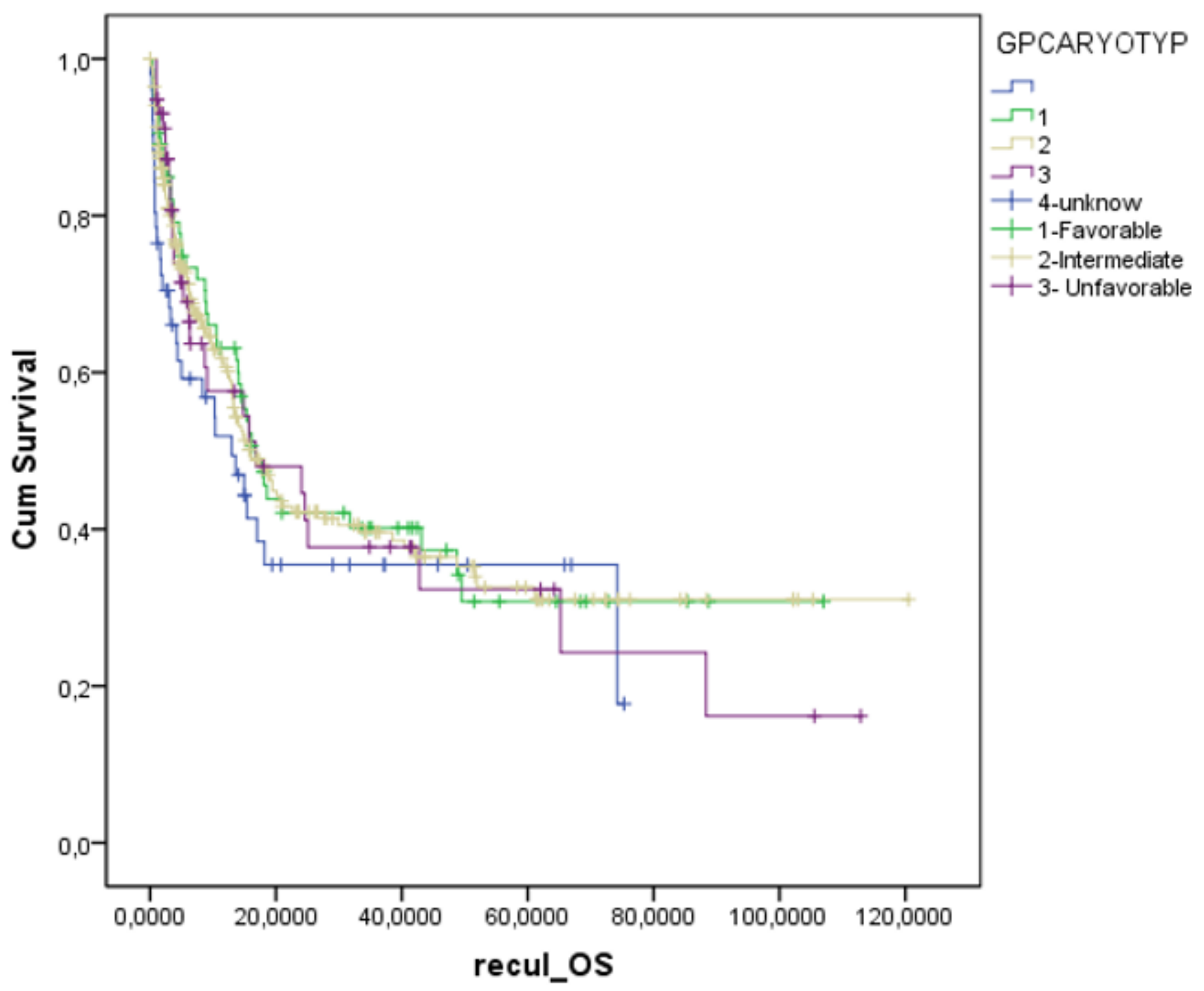

Figure 6

Overall survival of patients according to cytogenetic prognosis 